\title{
Isokibdelones: Novel Heterocyclic Polyketides from a
}

\section{Kibdelosporangium sp.}

\author{
Ranjala Ratnayake, Ernest Lacey, ${ }^{\dagger}$ Shaun Tennant,,$^{\dagger}$ Jennifer H. Gill ${ }^{\dagger}$ and Robert J. Capon. ${ }^{* *}$
}

Centre for Molecular Biodiversity, Institute for Molecular Bioscience, The University of Queensland, St

Lucia, Queensland, 4072, Australia and Microbial Screening Technologies Pty Ltd, Building A, 28-54

Percival Rd Smithfield, NSW 2164, Australia.

r.capon@imb.uq.edu.au

\section{Supporting Information-Experimental section}

General Experimental. Details are as reported previously. ${ }^{1}$

Fungal Collection and Identification. MST-108465 was isolated from a soil sample, collected from a timber woolshed $15 \mathrm{~km}$ north of Port Augusta in South Australia in 1996. Analysis of the non-polar secondary metabolites produced by the culture identified the presence of a series of metabolites with unusual UV spectra. A comparison of the UV spectra against a library of 1,500 microbial metabolites failed to identify any of the metabolites as known. A more extensive search of the UV spectra using extracts derived from Microbial Screening Technology's database of 6,000 type microbial species, failed to identify these metabolites as belonging to a known or characterized microbial species. ${ }^{2} 16 \mathrm{~S}$ rRNA analysis identified MST-108465 as belonging to the Pseudonocardiaceae and having 98\% identity with Kibdelosporangium spp. (aff. phillipinense). MST-108465 was found to share no common secondary metabolites with any known species of Kibdelosporangium. Accordingly, based on rRNA and metabolite data MST-108465 is regarded as a novel species, Kibdelosporangium sp. (MST-108465).

\section{Culturing and Processing.}


Mixed media fermentation of MST-108465: Fermentation details are similar to those reported for the kibdelones. $^{1}$

Fermentation of MST-108465 on wheat grain: In the mixed media fermentation, wheat was found to afford a different distribution of kibdelone-like metabolites to that observed on ISP2 agar. MST-108465 was recultured on wheat in an effort to produce sufficient amounts of these metabolites to enable characterization. The wheat fermentation $(20 \mathrm{x} 100 \mathrm{~g}$ wheat in $15 \mathrm{~cm}$ diameter Petri plates, incubated for 18 days at $28^{\circ} \mathrm{C}$ ) was extracted with EtOAc (3L).

\section{Isolation of isokibdelones}

(1) From mixed media culture: The individual extracts from the fermentation were pooled to provide $4 \mathrm{~L}$ of methanolic extract. This extract was concentrated in vacuo to an aqueous residue $(500 \mathrm{~mL})$ that was diluted with $\mathrm{H}_{2} \mathrm{O}$ to a final volume of $2 \mathrm{~L}$. This cloudy solution was passed through two parallel $\mathrm{C}_{18}$ solid phase extraction (SPE) cartridges $\left(2 \times 10 \mathrm{~g}\right.$, Varian $\mathrm{HF} \mathrm{C} \mathrm{C}_{18}$ ), eluting with 50\% aqueous $\mathrm{MeOH}(2 \times 80 \mathrm{~mL}$ each) followed by elution with $100 \% \mathrm{MeOH}(2 \times 80 \mathrm{~mL}$ each). HPLC and bioassay confirmed the presence of the activity and non-polar metabolites in the $\mathrm{MeOH}$ fraction. Partition of the pooled $\mathrm{MeOH}$ fraction (160 $\mathrm{mL})$ with hexane removed non-polar fats $(134 \mathrm{mg})$ yielding an enriched active methanol fraction $(875 \mathrm{mg})$.

The $\mathrm{MeOH}$ fraction was dissolved in a mixture of DMSO and methanol and fractionated by preparative HPLC (60 mL/min with gradient elution of $70 \%$ to $10 \% \mathrm{H}_{2} \mathrm{O} / \mathrm{MeCN}$ over $20 \mathrm{~min}$ followed by MeCN for $10 \mathrm{~min}$, through a $5 \mathrm{~mm}$ Platinum EPS $\mathrm{C}_{18} 50$ x $100 \mathrm{~mm}$ column). One hundred fractions were collected, concentrated in vacuo and combined on the basis of analytical HPLC analysis. A relatively non-polar pool of fractions $(13.7 \mathrm{mg})$ was concentrated in vacuo and further fractionated by HPLC $(2.5 \mathrm{~mL} / \mathrm{min}$ gradient elution from $50 \% \mathrm{H}_{2} \mathrm{O} / \mathrm{MeCN}$ to $100 \% \mathrm{MeCN}$ over 20 min through a Phenomenex LUNA $\mathrm{C}_{18} 5 \mu \mathrm{m}(2)$ $250 \times 10 \mathrm{~mm}$ column), to yield isokibdelone A (2) $(2.1 \mathrm{mg}, 0.24 \%$ yield). (NOTE: calculation of yield is based on the crude extract weight after hexane partition)

(2) From the Wheat grain fermentation: The EtOAc extract was washed with water and the organic layer concentrated in vacuo to yield a crude extract $(1.94 \mathrm{~g})$. The crude extract was dissolved in methanol, defatted with hexane and concentrated in vacuo to yield an enriched extract (1.44 g). The extract was subjected to preparative HPLC $\left(60 \mathrm{~mL} / \mathrm{min}\right.$ with gradient elution of $70 \%$ to $10 \% \mathrm{H}_{2} \mathrm{O} / \mathrm{MeCN}$ over 20 
minutes followed by $\mathrm{MeCN}$ for $10 \mathrm{~min}$, through a $5 \mathrm{~mm}$ Platinum $\mathrm{EPS} \mathrm{C}_{18} 50$ x $100 \mathrm{~mm}$ column). One hundred fractions were collected, concentrated in vacuo and combined on the basis of analytical HPLC analysis.

Two fractions containing "kibdelone-like" metabolites were identified by a combination of UV, ESI( $( \pm)$ MS and ${ }^{1} \mathrm{H}$ NMR spectroscopy and were subjected to multiple serial fractionation by HPLC (2.0 $\mathrm{mL} / \mathrm{min}$ gradient elution from $50-60 \% \mathrm{H}_{2} \mathrm{O} / \mathrm{MeCN}$ to $20 \% \mathrm{H}_{2} \mathrm{O} / \mathrm{MeCN}$ or $100 \% \mathrm{MeCN}$ over 20 min through a $\mathrm{C}_{8}$ $5 \mu \mathrm{m}$ Zorbax-StableBond $250 \times 9.4 \mathrm{~mm}$ column). This gave, in relative order of elution, isokibdelone A rhamnoside (2a; $3.3 \mathrm{mg} \mathrm{0.23 \% ),} \mathrm{isokibdelone} \mathrm{B} \mathrm{(3;} 0.6 \mathrm{mg} \mathrm{0.04 \% ),} \mathrm{and} \mathrm{isokibdelone} \mathrm{A} \mathrm{(2)} \mathrm{(3.1} \mathrm{mg,}$ $0.22 \%$ ). All yields are calculated relative to defatted enriched fraction (1.44 g). Isokibdelone C (4) was detected as minor constituents during the above purification process. The structure for $\mathbf{4}$ was proposed on the evidence based on LC-DAD-MS analysis and by comparison to the biosynthetically related kibdelone ${ }^{1}$ and isokibdelone analogues. 


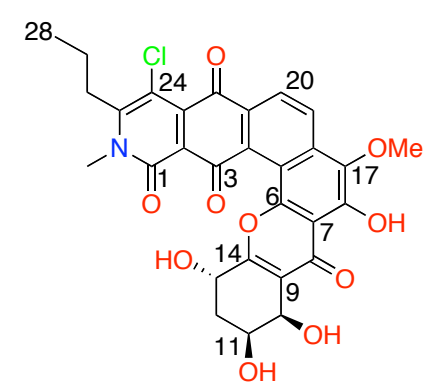

Isokibdelone A (2). Orange amorphous solid; $[\alpha]_{\mathrm{D}}+133^{\circ}\left(c 0.015, \mathrm{CHCl}_{3}\right)$; UV (EtOH) $\lambda_{\text {max }}\left(\varepsilon / \mathrm{dm}^{3} \mathrm{~mol}^{-1} \mathrm{~cm}^{-1}\right) 442$ (sh), 404 (11700), 319 (16300), 271 (26600), 239 (27600) nm; ${ }^{1} \mathrm{H}$ NMR and ${ }^{13} \mathrm{C}$ NMR $\left(\mathrm{CDCl}_{3}, 500 \mathrm{MHz}\right.$ and 100 $\mathrm{MHz}$ respectively); ESI(+)MS $m / z$ 582.0/584.0 $[\mathrm{M}+\mathrm{H}]^{+}$and ESI(-)MS $\mathrm{m} / z$ $580.1 / 582.0[\mathrm{M}-\mathrm{H}]$; $\mathrm{HRESI}(+) \mathrm{MS} 604.0982\left([\mathrm{M}+\mathrm{Na}]^{+}, \mathrm{C}_{29} \mathrm{H}_{24}{ }^{35} \mathrm{ClNO}_{10} \mathrm{Na}\right.$ requires 604.0986).

Table 1: NMR ( $\left.\mathrm{CDCl}_{3}, 500 \mathrm{MHz}\right)$ assignments for isokibdelone A (2)

\begin{tabular}{|c|c|c|c|c|}
\hline No. & $\begin{array}{llll}{ }^{1} \mathbf{H} & \delta_{\mathrm{H}}(\mathrm{m}, J(\mathrm{~Hz}))\end{array}$ & ${ }^{13} \mathrm{C}$ & COSY & НMBC \\
\hline 1 & & 158.6 & & \\
\hline 2 & & \# & & \\
\hline 3 & & $183.5^{\mathrm{b}}$ & & \\
\hline 4 & & $135.3^{\mathrm{c}}$ & & \\
\hline 5 & & 111.5 & & \\
\hline 6 & & $150.0^{\mathrm{a}}$ & & \\
\hline 7 & & 112.2 & & \\
\hline 8 & & $183.4^{\mathrm{b}}$ & & \\
\hline 9 & & 116.5 & & \\
\hline 10 & $5.04(\mathrm{brd}, 3.0)$ & 66.9 & $\mathrm{H}-11,10-\mathrm{OH}$ & C-14, C-9 \\
\hline 11 & 4.39 (brdd, 5.5, 3.0) & 66.5 & H-12a, H-12b, H-10 & $\mathrm{C}-10, \mathrm{C}-9$ \\
\hline $12 \mathrm{a}$ & $2.74(\mathrm{ddd}, 14.0,6.5,5.5)$ & 33.9 & H-13, H-12b, H-11 & $\mathrm{C}-14, \mathrm{C}-13, \mathrm{C}-11, \mathrm{C}-10$ \\
\hline $12 b$ & $1.82(\mathrm{ddd}, 14.0,9.0,2.0)$ & & $\mathrm{H}-13, \mathrm{H}-12 \mathrm{a}, \mathrm{H}-11$ & $\mathrm{C}-13$ \\
\hline 13 & $5.24(\mathrm{ddd}, 9.0,6.5,5.0)$ & 62.8 & $\mathrm{H}-12 \mathrm{a}, \mathrm{H}-12 \mathrm{~b}, 13-\mathrm{OH}$ & \\
\hline 14 & & 166.9 & & \\
\hline \multicolumn{5}{|l|}{$15[\mathrm{O}]$} \\
\hline 16 & & 149.7 & & \\
\hline 17 & & 136.6 & & \\
\hline 18 & & $135.3^{\mathrm{c}}$ & & \\
\hline 19 & $8.45(\mathrm{~d}, 9.0)$ & 126.3 & $\mathrm{H}-20$ & C-21 C-17 C-5 \\
\hline 20 & $8.23(\mathrm{~d}, 9.0)$ & 125.3 & H-19 & C- 22 C-18 C-4 \\
\hline 21 & & 131.4 & & \\
\hline 22 & & 181.1 & & \\
\hline 23 & & $138.8^{\mathrm{a}}$ & & \\
\hline 24 & & 108.9 & & \\
\hline 25 & & 155.9 & & \\
\hline 26 & $3.08(\mathrm{dd}, 8.5,8.0)$ & 33.8 & $\mathrm{H}-27$ & $\mathrm{C}-28, \mathrm{C}-27, \mathrm{C}-25, \mathrm{C}-24$ \\
\hline 27 & $1.74(\mathrm{~m})$ & 20.4 & H-28, H-26 & $\mathrm{C}-28, \mathrm{C}-25$ \\
\hline 28 & $1.21(\mathrm{t}, 7.5)$ & 14.2 & $\mathrm{H}-27$ & $\mathrm{C}-27, \mathrm{C}-26$ \\
\hline 17-OMe & $3.98(\mathrm{~s})$ & 61.1 & & $\mathrm{C}-17$ \\
\hline $\mathrm{NMe}$ & $3.74(\mathrm{~s})$ & 33.6 & & $\mathrm{C}-1$ \\
\hline $10 \mathrm{OH}$ & 4.88 (brs) & & $\mathrm{H}-10$ & $\mathrm{C}-10$ \\
\hline $11 \mathrm{OH}$ & & & & \\
\hline $13 \mathrm{OH}$ & $5.92(\mathrm{~d}, 5.0)$ & & $\mathrm{H}-13$ & $\mathrm{C}-12, \mathrm{C}-13$ \\
\hline $16 \mathrm{OH}$ & $12.68(\mathrm{~s})$ & & & $\mathrm{C}-17, \mathrm{C}-16, \mathrm{C}-7$ \\
\hline
\end{tabular}

Isokibdelone B (3). Orange amorphous solid $[\alpha]_{\mathrm{D}}+82^{\circ}\left(c 0.02, \mathrm{CHCl}_{3}\right)$; UV (EtOH) $\lambda_{\max }\left(\varepsilon / \mathrm{dm}^{3} \mathrm{~mol}^{-1} \mathrm{~cm}^{-1}\right) 583(\mathrm{sh}), 447$ (3700), $326(\mathrm{sh}), 266(\mathrm{sh}), 244$ 
(9500), 203 (13700) nm; ${ }^{1} \mathrm{H}$ NMR and ${ }^{13} \mathrm{C}$ NMR ( $d_{6}$-DMSO, $600 \mathrm{MHz}$ and $150 \mathrm{MHz}$ respectively), see Table 8; ESI(+)MS m/z 584.0/586.2 [M+H] $]^{+}$and ESI(-)MS $m / z$ 582.1/584.0 [M-H]; HRESI(+)MS $606.1133\left([\mathrm{M}+\mathrm{Na}]^{+} \mathrm{C}_{29} \mathrm{H}_{26}{ }^{35} \mathrm{ClNO}_{10} \mathrm{Na}\right.$ requires 606.1143).

Table 2: NMR $\left(d_{6}\right.$-DMSO, $\left.600 \mathrm{MHz}\right)$ assignments for isokibdelone B (3)

\begin{tabular}{|c|c|c|c|c|}
\hline No. & $\begin{array}{llll}{ }^{1} \mathbf{H} & \delta_{\mathrm{H}}(\mathrm{m}, J(\mathbf{H z}))\end{array}$ & ${ }^{13} \mathrm{C}$ & COSY & HMBC \\
\hline 1 & & 157.0 & & \\
\hline 2 & & $119.2^{\mathrm{a}}$ & & \\
\hline 3 & & $181.8^{\mathrm{a}, \mathrm{b}}$ & & \\
\hline 4 & & $141.3^{\mathrm{a}, \mathrm{c}}$ & & \\
\hline 5 & & $106.8^{\mathrm{a}}$ & & \\
\hline 6 & & $148.3^{\mathrm{a}}$ & & \\
\hline 7 & & 109.1 & & \\
\hline 8 & & 182.4 & & \\
\hline 9 & & 116.8 & & \\
\hline 10 & $4.66(\mathrm{brd}, 3.3)$ & 57.9 & $\mathrm{H}-12 \mathrm{~b}, \mathrm{H}-11,10-\mathrm{OH}$ & $\mathrm{C}-14, \mathrm{C}-13, \mathrm{C}-12, \mathrm{C}-9, \mathrm{C}-8$ \\
\hline 11 & $3.86(\mathrm{ddd}, 12.5,3.3,3.2)$ & 68.7 & $\mathrm{H}-12 \mathrm{a}, \mathrm{H}-12 \mathrm{~b}, \mathrm{H}-10$ & \\
\hline $12 \mathrm{a}$ & $2.20(\mathrm{ddd}, 13.2,12.5,4.5)$ & 30.9 & $\mathrm{H}-13, \mathrm{H}-12 \mathrm{~b}, \mathrm{H}-11$ & $C-13, C-10$ \\
\hline $12 b$ & $1.75(\mathrm{brd}, 13.2)$ & & $\mathrm{H}-13, \mathrm{H}-12 \mathrm{a}, \mathrm{H}-11$ & $\mathrm{C}-14, \mathrm{C}-13, \mathrm{C}-10$ \\
\hline 13 & 4.48 (brd, 4.5$)$ & 64.8 & $\mathrm{H}-12$ & $\mathrm{C}-14$ \\
\hline 14 & & 165.4 & & \\
\hline \multicolumn{5}{|l|}{$15[\mathrm{O}]$} \\
\hline 16 & & 154.8 & & \\
\hline 17 & & 139.7 & & \\
\hline 18 & & $139.45^{\mathrm{a}, \mathrm{c}}$ & & \\
\hline \multirow[t]{2}{*}{19} & $2.74(\mathrm{~m})$ & 18.2 & $\mathrm{H}-20$ & \\
\hline & $2.63(\mathrm{~m})$ & & & \\
\hline \multirow[t]{2}{*}{20} & $2.97(\mathrm{~m})$ & 21.3 & H-19 & \\
\hline & $2.86(\mathrm{~m})$ & & & \\
\hline 21 & & $139.4^{\mathrm{a}, \mathrm{c}}$ & & \\
\hline 22 & & $181.1^{\mathrm{a}, \mathrm{b}}$ & & \\
\hline 23 & & $137.8^{\mathrm{a}, \mathrm{c}}$ & & \\
\hline 24 & & 108.9 & & \\
\hline 25 & & 157.5 & & \\
\hline 26 & $3.05(\mathrm{ddd}, 8.3,8.2,2.6)$ & 33.2 & H-27 & $\mathrm{C}-28, \mathrm{C}-27, \mathrm{C}-25, \mathrm{C}-24$ \\
\hline 27 & $1.64(\mathrm{~m})$ & 19.6 & H-28 H-26 & C-28, C-26, C-25 \\
\hline 28 & $1.06(t, 7.3)$ & 13.9 & H-27 & $\mathrm{C}-27, \mathrm{C}-26$ \\
\hline $17-\mathrm{OMe}$ & $3.83(\mathrm{~s})$ & 60.2 & & $\mathrm{C}-17$ \\
\hline $\mathrm{NMe}$ & $3.71(\mathrm{~s})$ & 33.5 & & C-1 \\
\hline \multicolumn{5}{|l|}{$10 \mathrm{OH}$} \\
\hline \multicolumn{5}{|l|}{$11 \mathrm{OH}$} \\
\hline $13 \mathrm{OH}$ & $5.45(\mathrm{brs})$ & & H-13 & \\
\hline $16 \mathrm{OH}$ & $13.32(\mathrm{~s})$ & & & $\mathrm{C}-17, \mathrm{C}-16, \mathrm{C}-7$ \\
\hline
\end{tabular}

${ }^{a}$ Assignments are made in comparison with literature data for reported compounds and biosynthetic analogues from same culture. ${ }^{\text {b,c }}$ values may be interchanged

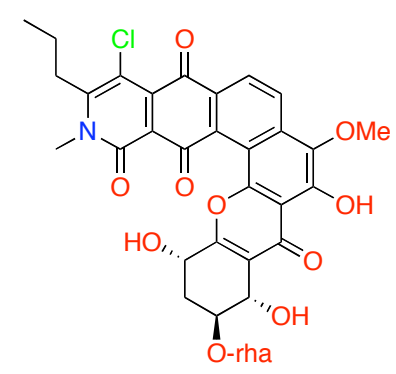

Isokibdelone A rhamnoside (2a). Orange amorphous solid $[\alpha]_{\mathrm{D}}+60^{\circ}(c$ $\left.0.015, \mathrm{CHCl}_{3}\right) ; \mathrm{UV}(\mathrm{EtOH}) \lambda_{\max }\left(\varepsilon / \mathrm{dm}^{3} \mathrm{~mol}^{-1} \mathrm{~cm}^{-1}\right) 442(\mathrm{sh}), 410$ (6700), 271 (17400), 229 (23200), 210 (25000) nm; ${ }^{1} \mathrm{H}$ NMR and ${ }^{13} \mathrm{C}$ NMR $\left(d_{6}\right.$-DMSO, $600 \mathrm{MHz}$ and $150 \mathrm{MHz}$ respectively); $\mathrm{ESI}(+) \mathrm{MS} \mathrm{m} / \mathrm{z}$ 728.2/730.3 [M+H] , 
582.2/584.2 (M-rha+H) and ESI(-)MS m/z 726.4/728.0 [M-H] ; HRESI(+)MS $750.1569\left([\mathrm{M}+\mathrm{Na}]^{+}\right.$, $\mathrm{C}_{35} \mathrm{H}_{34}{ }^{35} \mathrm{ClNO}_{14} \mathrm{Na}$ requires 750.1566$)$.

Table 3: NMR ( $d_{6}$-DMSO, $\left.600 \mathrm{MHz}\right)$ assignments for isokibdelone A-rhamnoside (2a)

\begin{tabular}{|c|c|c|c|c|}
\hline No. & $\begin{array}{lll}{ }^{1} \mathbf{H} & \delta_{\mathrm{H}}(\mathrm{m}, J(\mathbf{H z}))\end{array}$ & ${ }^{13} \mathrm{C}$ & COSY & НМBC \\
\hline 1 & & 157.6 & & \\
\hline 2 & & $121.7^{\mathrm{a}}$ & & \\
\hline 3 & & $183.4^{\mathrm{a}}$ & & \\
\hline 4 & & 134.2 & & \\
\hline 5 & & 110.8 & & \\
\hline 6 & & $149.3^{\mathrm{a}}$ & & \\
\hline 7 & & 117.7 & & \\
\hline 8 & & 182.2 & & \\
\hline 9 & & 118.2 & & \\
\hline 10 & 4.97 (brs) & 57.9 & $\mathrm{H}-12 \mathrm{~b}, \mathrm{H}-11,10-\mathrm{OH}$ & $\mathrm{C}-14, \mathrm{C}-11, \mathrm{C}-9, \mathrm{C}-8$ \\
\hline 11 & $4.03(\mathrm{ddd}, 12.5,3.2,3.1)$ & 68.7 & $\mathrm{H}-10, \mathrm{H}-12 \mathrm{a}, \mathrm{H}-12 \mathrm{~b}$ & $\mathrm{C}-1^{\prime}$ \\
\hline $12 \mathrm{a}$ & $2.43(\mathrm{ddd}, 13.3,12.5,5.2)$ & 30.9 & $\mathrm{H}-13, \mathrm{H}-12 \mathrm{~b}, \mathrm{H}-11$ & $\mathrm{C}-11$ \\
\hline $12 \mathrm{~b}$ & $1.86(\mathrm{brd}, 13.3)$ & & H-13, H-12a, H-11 & $\mathrm{C}-14, \mathrm{C}-13, \mathrm{C}-11, \mathrm{C}-10$ \\
\hline 13 & $4.76(\mathrm{dd}, 5.2,5.0)$ & 64.2 & $\mathrm{H}-12$ & C-14 \\
\hline 14 & & 165.4 & & \\
\hline \multicolumn{5}{|l|}{$15[\mathrm{O}]$} \\
\hline 16 & & 149.1 & & \\
\hline 17 & & 135.9 & & \\
\hline 18 & & 135.6 & & \\
\hline 19 & $8.35(\mathrm{~d}, 9.0)$ & 125.5 & H-20 & C-21, C-17, C-5 \\
\hline 20 & $8.16(d, 9.0)$ & 125.1 & H-19 & C-22, C-18, C-4 \\
\hline 21 & & 130.8 & & \\
\hline 22 & & 180.6 & & \\
\hline 23 & & $138.4^{\mathrm{a}}$ & & \\
\hline 24 & & 107.0 & & \\
\hline 25 & & 156.7 & & \\
\hline 26 & $3.06(\mathrm{dd}, 8.3,8.2)$ & 33.1 & $\mathrm{H}-27$ & $\mathrm{C}-28, \mathrm{C}-27, \mathrm{C}-25, \mathrm{C}-24$ \\
\hline 27 & $1.65(\mathrm{~m})$ & 19.7 & H-28 H-26 & $\mathrm{C}-28, \mathrm{C}-26, \mathrm{C}-25$ \\
\hline 28 & $1.07(\mathrm{t}, 7.3)$ & 13.9 & $\mathrm{H}-27$ & C-27, C-26 \\
\hline 17-OMe & $3.98(\mathrm{~s})$ & 60.7 & & C-17 \\
\hline $\mathrm{NMe}$ & $3.74(\mathrm{~s})$ & 33.7 & & C-1 \\
\hline $10 \mathrm{OH}$ & $5.28(\mathrm{brs})$ & & H-12 & \\
\hline \multicolumn{5}{|l|}{$11 \mathrm{OH}$} \\
\hline $13 \mathrm{OH}$ & $5.52(\mathrm{~d}, 5.0)$ & & H-13 & C-12 \\
\hline $16 \mathrm{OH}$ & $12.92(\mathrm{~s})$ & & & C-17, C-16, C-7 \\
\hline $1^{\prime}$ & 4.91 (brs) & 97.9 & H-2' & $\mathrm{C}-5^{\prime}, \mathrm{C}-3^{\prime}$ \\
\hline $2^{\prime}$ & $3.75(\mathrm{dd}, 1.5,3.0)$ & 70.4 & H-3', H-1' & C-4,, C-3 \\
\hline $3^{\prime}$ & $3.54(\mathrm{dd}, 3.0,9.5)$ & 70.6 & $\mathrm{H}-4^{\prime}, \mathrm{H}-2^{\prime}$ & $\mathrm{C}-4^{\prime}$ \\
\hline $4^{\prime}$ & $3.24(\mathrm{dd}, 9.5,9.3)$ & 72.1 & $\mathrm{H}-5^{\prime}, \mathrm{H}-3^{\prime}$ & $\mathrm{C}-6^{\prime}, \mathrm{C}-5^{\prime}, \mathrm{C}-3^{\prime}, \mathrm{C}-2^{\prime}$ \\
\hline $5^{\prime}$ & 3.52 (brd, 9.3) & 68.9 & H-6', H-4 & $\mathrm{C}-4^{\prime}$ \\
\hline $6^{\prime}$ & $1.18(\mathrm{~d}, 6.3)$ & 17.9 & $\mathrm{H}-5^{\prime}$ & $\mathrm{C}-5^{\prime}, \mathrm{C}-4^{\prime}$ \\
\hline
\end{tabular}

\section{Biological activity}

Anticancer assays were carried out as detailed previously for the kibdelones. ${ }^{1}$ Procedures for antimicrobial, nematocidal and NS-1 cytotoxicity have been described previously. ${ }^{3}$

\section{Reference:}

1. $\quad$ Ratnayake, R.; Lacey, E.; Tennant, S.; Gill, J. H.; Capon, R. J. Chem. Eur. J. 2006 accepted.

2. Lacey, E.; Tennant, S. Microbiol. Australia 2003, 24, 34-35. 
3. Capon, R. J.; Ratnayake, R.; Stewart, M.; Lacey, E.; Tennant, S.; Gill, J. H. Org. Biomol. Chem. 2005, 3, 123129. 